\title{
Personalien
}

Todesfälle / Décès / Decessi

Erwin A. Koller (1933), † 1.8.2015, 8057 Zürich

Georges Muheim (1938), † 20.7.2015, Spécialiste en chirurgie orthopédique et traumatologie de l'appareil locomoteur, 1700 Fribourg

Erika Elfriede H. Gold (1923), † 30.6.2015, 8008 Zürich

Otto Soyka (1918), † 31.7.2015,

Facharzt für Allgemeine Innere Medizin, 8052 Zürich

Rudolf Ausfeld (1947), † 8.7.2015,

Facharzt für Urologie, 5032 Bibernstein

Zeljko Stjepan Sojcic (1958), † 26.7.2015, Facharzt für Allgemeine Innere Medizin, 3027 Bern
Ärztegesellschaft des Kantons Luzern

Zur Aufnahme in unsere Gesellschaft Sektion Stadt haben sich gemeldet:

Karl Georg Heinrich, Praktischer Arzt, AZ Swissclinic AG, Pilatusstrasse 35, 6003 Luzern

Friedrich Christian Gönner, Facharzt für Diagnostische Radiologie, FMH,

ab 1.10.2015 Radiologie Luzern AG, Zihlmattweg 46, 6005 Luzern

Arto Christian Nirkko, Facharzt für Neurologie, FMH, Klinik für Schlafmedizin KSM, Lützelmattstrasse 3, 6006 Luzern

Lars Meierkord, Facharzt Orthopädie und Traumatologie des Bewegungsapparates, SUVA Zentralschweiz, Versicherungsmedizin, Löwenplatz 1, 6002 Luzern

Einsprachen sind innert 20 Tagen nach der Publikation schriftlich und begründet zu richten an: Ärztegesellschaft des Kantons Luzern, Schwanenplatz 7, 6004 Luzern.

\section{Ärztegesellschaft Thurgau}

Zum Eintritt in die Ärztegesellschaft Thurgau haben sich gemeldet:

Regine Werner, Säntisstrasse 6, 8556 Illhart

Michaela Lentz, Mittlerer Rafensburgweg 1, 8274 Tägerwilen 